\title{
High Efficiency Protein Extraction from Adipose Tissues
}

Invent Biotechnologies Inc.

\section{Introduction}

Proteomic research using adipose tissues are increasingly common. It is relatively easy to extract total proteins from most cell types and tissues however it is much more difficult to extract high quality total proteins from adipose tissues. A common feature shared by adipose tissues is their high lipid and low protein content. In average, the protein content accounts for less than $2 \%$ of total cellular mass, making it technically very challenging to isolate high concentration total proteins from adipose tissues [1]. Here we report a high efficiency total protein extraction method from adipose tissues.

\section{Materials and Method}

Total proteins were extracted from white adipose tissues of porcine, chicken, and rat using Minute ${ }^{T M}$ Total Protein Extraction Kit for Adipose Tissues/ Cultured Adipocytes (Invent Biotechnologies, Plymouth, MN, USA. Cat.\# AT-022) according to manufacturer's instruction. Briefly, adipose tissues were homogenized in hypotonic buffer in an Eppendorf tube. The cell lysates containing water-oil emulsion were incubated at $-20^{\circ} \mathrm{C}$. After incubation, the aggregated lipid in the cell lysates were separated by passing through a special filter cartridge. The pass through containing total cellular protein was analyzed by SDS-PAGE and Western blotting.

\section{Results and Discussion}

As shown in Figure 1, total protein was extracted from white adipose tissues of porcine (A1), chicken (A2) and rat (A3). The proteins cover the whole spectrum in size distribution ranging from $>250 \mathrm{KD}$ to $<30 \mathrm{KD}$ indicating high extraction efficiency. Subsequent Western blotting results using rat fat tissue further confirms the completeness of extracted proteins (B). Subcellular compartment markers for plasma membrane (Na/K-ATPase), mitochondrial (ubiquinol-cytochrome C), cytosolic (GAPDH) and nuclear (Lamin-B1) proteins are readily detectable using colorimetric substrate. The protein yield is high (2-3 mg/ml). The performance of this new technology is also confirmed by a recent publication [2] for the study of phosphoproteins of adipose tissues. RIPA buffer has been widely used for extracting proteins from adipose tissues. However, the quality of extracted protein in many cases is far from satisfactory. Detergents present in RIPA and other extraction buffer frequently leads to protein sequestration into micelles, resulting in protein loss and poor yield [3]. In order to overcome the disadvantages of detergent-containing extraction buffers, several detergent-free and organic solvent-based protein extraction protocols have also been developed [1], however, these methods are relatively tedious and time consuming. Because detergent is not used in the extraction process, it is not clear if a complete protein profile can be obtained. The protein yield is also low with these methods $(0.5-1 \mathrm{mg} / \mathrm{ml})$.

More information on protein extraction and nuclear/cytosolic fractionation of adipose tissues can be found at www.inventbiotech.com

\section{References}

1. Sajic, T. et al. (2011) Analytical Chem. 415:215-217.

2. Wang, N. et al. (2017). Life Sciences. doi:10.1016/j.lfs.2017.08.023

3. Pokhariyal, R. et al. (2014) J. Proteins and Proteomics 5:121-124. 